\section{(O) OPEN ACCESS}

\title{
High rates of non-adherence to antihypertensive treatment revealed by high-performance liquid chromatography-tandem mass spectrometry (HP LC-MS/MS) urine analysis
}

\author{
Maciej Tomaszewski, ${ }^{1,2}$ Christobelle White, ${ }^{1,2}$ Prashanth Patel, ${ }^{3}$ Nicholas Masca, ${ }^{1,2}$ \\ Ravi Damani, ${ }^{1}$ Joanne Hepworth, ${ }^{3}$ Nilesh J Samani, ${ }^{1,2}$ Pankaj Gupta, ${ }^{1,3}$ \\ Webster Madira, ${ }^{3}$ Adrian Stanley, ${ }^{1,3}$ Bryan Williams $^{4}$
}

\begin{abstract}
${ }^{1}$ Department of Cardiovascular Sciences, University of Leicester, Leicester, UK ${ }^{2} \mathrm{NIHR}$ Leicester Biomedical Research Unit in

Cardiovascular Disease, Leicester, UK

${ }^{3}$ Department of Chemical Pathology, University Hospitals of Leicester NHS Trust, Leicester, UK

${ }^{4}$ Institute of Cardiovascular Science and NIHR University College London Hospitals Biomedical Research Centre, University College London, London, UK
\end{abstract}

\section{Correspondence to} Dr Maciej Tomaszewski, Department of Cardiovascular Sciences, University of Leicester, Clinical Sciences Wing, Glenfield Hospital, Leicester, LE3 9QP, UK; mt142@le.ac.uk

Received 7 October 2013 Revised 7 January 2014 Accepted 14 January 2014 Published Online First 3 April 2014

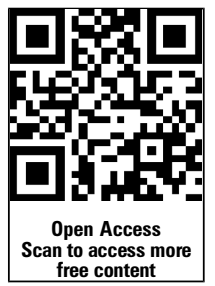

\section{SLinked}

- http://dx.doi.org/10.1136/ heartjnl-2014-305540

To cite: Tomaszewski $\mathrm{M}$ White $C$, Patel $\mathrm{P}$, et al. Heart 2014;100:855-861.

\section{ABSTRACT}

Objectives Non-adherence to therapy is an important cause of suboptimal blood pressure control but few practical tools exist to accurately and routinely detect it. We used a simple urine-based assay to evaluate the prevalence of antihypertensive treatment non-adherence and its impact on blood pressure in a specialist hypertension centre.

Methods 208 hypertensive patients (125 new referrals, 66 follow-up patients with inadequate blood pressure control and 17 renal denervation referrals) underwent assessment of antihypertensive drug intake using highperformance liquid chromatography-tandem mass spectrometry (HP LC-MS/MS) urine analysis at the time of clinical appointment. A total of 40 most commonly prescribed antihypertensive medications (or their metabolites) were screened for in spot urine samples.

Results Overall, $25 \%$ of patients were totally or partially non-adherent to antihypertensive treatment (total non-adherence $10.1 \%$, partial non-adherence $14.9 \%)$. The highest prevalence of partial and total nonadherence was among follow-up patients with inadequate blood pressure control $(28.8 \%)$ and those referred for consideration of renal denervation $(23.5 \%)$, respectively. There was a linear relationship between blood pressure and the numerical difference in detected/ prescribed antihypertensive medications-every unit increase in this difference was associated with 3.0 (1.1) $\mathrm{mm} \mathrm{Hg}, 3.1$ (0.7) $\mathrm{mm} \mathrm{Hg}$ and 1.9 (0.7) $\mathrm{mm} \mathrm{Hg}$ increase in adjusted clinic systolic blood pressure, clinic diastolic blood pressure (DBP) and $24 \mathrm{~h}$ mean daytime DBP $\left(p=0.0051, p=8.62 \times 10^{-6}, p=0.0057\right)$, respectively.

Conclusions Non-adherence to blood pressure lowering therapy is common, particularly in patients with suboptimal blood pressure control and those referred for renal denervation. HP LC-MS/MS urine analysis could be used to exclude non-adherence and better stratify further investigations and intervention.

Non-adherence to pharmacological treatment is recognised as an important barrier to the successful management of many chronic disorders, including hypertension. ${ }^{1}$ Poor concordance with treatment is considered to be one of the major causes of inadequate response to blood pressure (BP) lowering treatment in patients diagnosed with 'resistant hypertension' (up to $10 \%-20 \%$ of patients with high BP). ${ }^{2}$ This issue has recently come to the fore because of the availability of new non-drug therapies, that is, percutaneous radiofrequency, catheter-based renal sympathetic denervation (renal denervation) for patients with suspected resistance to antihypertensive treatment. ${ }^{2}{ }_{3}^{3}$ Furthermore, patients with undeclared/ unrecognised non-adherence frequently undergo numerous additional (sometimes invasive and often expensive) diagnostic tests in specialist centres to identify causes of their apparent poor response to antihypertensive medications. Moreover, patients who are non-adherent to antihypertensive treatment fail to gain the proven benefits of BP lowering therapy and remain at high risk of cardiovascular events. ${ }^{45}$

The true prevalence of non-adherence to antihypertensive treatment is not known. The published data suggest that poor adherence may be more common than full adherence to antihypertensive therapy. ${ }^{5} 6$ The estimates of prevalence differ across studies with non-adherence rates reported from as low as $3 \%$ and up to $65 \% .^{7}$ One of the reasons for this inconsistency is lack of direct and objective methods to screen for and monitor non-adherence to treatment among hypertensive patients. Furthermore, demographic and clinical characteristics (such as age, race, ethnicity, education, attendance for clinical appointments, duration of hypertension, coexistent comorbidities) are not or only very poor correlates of adherence and cannot provide accurate prediction of non-adherent behaviour. ${ }^{18}$ Clinical judgement is believed to overestimate the rate of non-adherence to antihypertensive treatment. ${ }^{9}$ Electronic methods of adherence monitoring (ie, computerised records of pharmacy prescription refills, electronic records of pill box opening or medication event monitoring systems) may provide some insight into long-term persistence with treatment and accurate timing of drug administration. They were used in studies on antihypertensive treatment. ${ }^{10}{ }^{11}$ However, as heavily dependent on patients' behaviour (ie, opening a box with medication may not always lead to its administration in patients who choose to conceal it), they are also imperfect proxies of adherence to therapy. 
Here we report the data from high-performance liquid chromatography-tandem mass spectrometry (HP LC-MS/MS) analysis of spot urine samples to detect a wide range of most commonly prescribed antihypertensive medications in $>200$ hypertensive patients attending a specialist clinical hypertension centre (Leicester, UK). Apart from patients whose response to clinic-prescribed BP lowering medications was suboptimal (suspected non-adherence), we also examined new referrals from primary care, as well as those referred for consideration of renal denervation.

\section{METHODS}

\section{Patients}

A total of 208 hypertensive patients who underwent HP LC-MS/MS biochemical screening for non-adherence to antihypertensive treatment at the Leicester BP centre between December 2011 and January 2013 were evaluated in this project. They were divided into three separate groups (A, B and C). A total of 125 individuals were new referrals from primary care (Group A). They were screened for non-adherence to antihypertensive therapy during their first appointment in the clinic. The only inclusion criterion of new patients in this project was a referral letter from primary care. Suboptimal BP control was one of the main but not the exclusive reason why patients from Group A were referred to the clinic. Group B consisted of 66 patients whose BP response to clinic-prescribed antihypertensive treatment was considered inadequate by a specialist in hypertension. All patients from Group B were under follow-up of the centre and prior to inclusion into the study they had been reviewed at least once in the clinic (on average five clinical appointments prior to screening). Included in Group C were 17 hypertensive patients formally referred to our centre for consideration of renal denervation. Their screening for adherence was a part of a thorough diagnostic work-up prior to consideration of this procedure. All patients in Group C had suboptimal BP response to treatment based on clinical assessment of the referring clinician. The patients referred for renal denervation were reviewed at least once in the clinic before test for therapeutic non-adherence (average number of previous appointments- 7).

Basic demographic information (ie, age, sex, ethnicity) were retrieved retrospectively from clinical files. Last seated clinic BP values, measured according to the National Institute of Clinical Excellence clinical guideline 127 using a validated semiautomatic device (A\&D Digital BP Monitor UA-767PC, A\&D Instruments) were recorded, prior to biochemical analysis of a spot urine sample. Information on clinic BP was available for 204 patients. Information on $24 \mathrm{~h}$ ambulatory mean daytime BP measured on the non-dominant arm (Spacelabs 90217A-1, Spacelabs Healthcare) was available for 147 patients.

\section{Urine analysis-HP LC-MS/MS}

Spot urine samples for further biochemical testing were collected in the morning on the day of the clinical appointment. The samples were transferred from the clinic to the laboratory at room temperature and stored at $-70^{\circ} \mathrm{C}$ until further analysis. Samples were prepared by solvent extraction and by dilution technique. The dilution technique was used to detect strongly polar drugs (such as lisinopril, nicorandil) that are difficult to extract. The samples were diluted in 1:10 in distilled water before HP LC-MS/MS. Weekly acidic, basic and neutral antihypertensive drugs (such as bisoprolol, diltiazem, amiloride) underwent solvent extraction. In the extraction process, $5 \mathrm{~mL}$ of urine was mixed with organic solvent for $10 \mathrm{~min}$. It was then centrifuged at $3000 \mathrm{rpm}$ for $5 \mathrm{~min}$. The upper organic layer was
Table 1 Antihypertensive medications and/or their metabolites examined in urine by high-performance liquid chromatography-tandem mass spectrometry

\begin{tabular}{|c|c|c|}
\hline No & Antihypertensive medication (metabolites) & Ion mode \\
\hline 1 & Enalapril (enalaprilat) & Positive \\
\hline 2 & Lisinopril & Positive \\
\hline 3 & Perindopril (perindoprilat) & Positive \\
\hline 4 & Ramipril (ramiprilat) & Positive \\
\hline 5 & Quinalapril (qunilaprilat) & Positive \\
\hline 6 & Trandolapril (trandolaprilat) & Positive \\
\hline 7 & Candesartan (desethylcandesartan) & Positive \\
\hline 8 & Irbesartan (hydroxyirbesartan) & Positive \\
\hline 9 & Valsartan (hydroxyvalsartan) & Positive \\
\hline 10 & Losartan (losartan acid) & Positive \\
\hline 11 & Telmisartan & Positive \\
\hline 12 & Olmesartan & Positive \\
\hline 13 & Atenolol & Positive \\
\hline 14 & Metoprolol & Positive \\
\hline 15 & Propranolol & Positive \\
\hline 16 & Labetolol & Positive \\
\hline 17 & Bisoprolol & Positive \\
\hline 18 & Nebivolol (hydroxynebivolol) & Positive \\
\hline 19 & Amlodipine & Positive \\
\hline 20 & Felodipine & Positive \\
\hline 21 & Lercanidipine & Positive \\
\hline 22 & Lacidipine & Positive \\
\hline 23 & Nifedipine (hydroxynifedipinic acid, nifedipinic acid) & Negative \\
\hline 24 & Diltiazem & Positive \\
\hline 25 & Verapamil (norverapamil) & Positive \\
\hline 26 & Bendroflumethiazide & Negative \\
\hline 27 & Hydrochlorothiazide & Negative \\
\hline 28 & Indapamide & Positive \\
\hline 29 & Furosemide & Negative \\
\hline 30 & Chlorthalidone & Positive \\
\hline 31 & Bumetanide & Positive \\
\hline 32 & Eplerenone & Positive \\
\hline 33 & Spironolactone (canrenone) & Positive \\
\hline 34 & Amiloride & Positive \\
\hline 35 & Hydralazine & Positive \\
\hline 36 & Doxazosin (hydroxydoxazosin) & Positive \\
\hline 37 & Prazosin & Positive \\
\hline 38 & Moxonidine (dehydromoxonidine) & Positive \\
\hline 39 & Aliskiren & Positive \\
\hline 40 & Methyldopa & Positive \\
\hline
\end{tabular}

evaporated under nitrogen at $40^{\circ} \mathrm{C}$ and reconstituted in $1 \mathrm{~mL}$ of $5 \%$ methanol prior to HP LC-MS/MS. Screening for lacidipine and nebivolol required a prior hydrolysis step $(5 \mathrm{~mL}$ of urine incubated with $200 \mu \mathrm{L}$ of $\beta$ glucuronidase for $2 \mathrm{~h}$ at $60^{\circ} \mathrm{C}$ ) before further analysis.

HP LC-MS/MS was performed using an Agilent Technologies 1290 series High Pressure Liquid Chromatograph interfaced with an Agilent Technologies 6460 Triple Quad Mass Spectrometer fitted with a Jetstream electrospray (ESI) source. The nebuliser gas temperature was set at $350^{\circ} \mathrm{C}$ with a flow of $5 \mathrm{~L} / \mathrm{min}$ and a pressure of 45 psi. The sheath gas temperature was set at $250^{\circ} \mathrm{C}$ and a flow of $11 \mathrm{~L} / \mathrm{min}$. The HP LC system was operated in gradient mode using $0.1 \%$ acetic acid in water 
Table 2 General demographic and clinical characteristics of hypertensive patients

\begin{tabular}{|c|c|c|c|c|}
\hline Phenotype & All & Group A & Group B & Group C \\
\hline $\mathrm{n}$ & 208 & 125 & 66 & 17 \\
\hline Age (years) & $56.7 \pm 16.0$ & $55.5 \pm 17.8$ & $57.4 \pm 13.4$ & $62.7 \pm 8.4$ \\
\hline $\operatorname{Sex}(M / F)$ & $93 / 115$ & $57 / 68$ & $28 / 38$ & $8 / 9$ \\
\hline BMI $\left(\mathrm{kg} / \mathrm{m}^{2}\right)^{*}$ & $31.5 \pm 6.8$ & $31.4 \pm 7.2$ & $31.6 \pm 6.3$ & $31.9 \pm 6.0$ \\
\hline White European ethnicity & $151(72.6)$ & $93(74.4)$ & $44(66.7)$ & $14(82.4)$ \\
\hline Clinical appointments before screening & $5(3-7) \dagger$ & - & $5(2-10)$ & $7(4-13)$ \\
\hline Clinic SBP $(\mathrm{mm} \mathrm{Hg}) \ddagger$ & $163 \pm 24.5$ & $160 \pm 24.5$ & $168 \pm 24.3$ & $168 \pm 22.1$ \\
\hline Clinic DBP $(\mathrm{mm} \mathrm{Hg}) \ddagger$ & $92 \pm 16.3$ & $90 \pm 15.1$ & $95 \pm 18.8$ & $94 \pm 13.3$ \\
\hline $24 \mathrm{~h}$ daytime SBP $(\mathrm{mm} \mathrm{Hg}) \S$ & $154 \pm 20.1$ & $151 \pm 19.7$ & $160 \pm 19.5$ & $160 \pm 21.0$ \\
\hline $24 \mathrm{~h}$ daytime $\mathrm{DBP}(\mathrm{mm} \mathrm{Hg}) \S$ & $87 \pm 13.4$ & $86 \pm 13.3$ & $91 \pm 13.4$ & $89 \pm 13.5$ \\
\hline
\end{tabular}

Group A-new referrals, Group B-follow-up patients with inadequate blood pressure control, Group C—patients referred for renal denervation.

*Body mass index (BMI)—data available for 185 patients.

†Average values for Groups B and C only, data are counts (number of individuals, sex) or counts and percentages (white European ethnicity), means and SDs (age, BMI, clinic blood

pressures, $24 \mathrm{~h}$ daytime blood pressures) or median and $25 \%-75 \%$ quartiles (clinical appointments before screening).

$¥$ Clinic systolic blood pressure (SBP) and diastolic blood pressure (DBP)—data available for 204 patients.

$\S 24 \mathrm{~h}$ daytime SBP and DBP—data available for 147 patients.

for mobile phase A and $0.1 \%$ acetic acid in methanol for mobile phase B. The initial conditions of $5 \% \mathrm{~B} / 95 \%$ A were held for $2 \mathrm{~min}$ and then ramped to $60 \% \mathrm{~B}$ at $6 \mathrm{~min}$ and further $100 \% \mathrm{~B}$ at $9 \mathrm{~min}$. The gradient was held at $100 \% \mathrm{~B}$ for $1 \mathrm{~min}$ and then returned to $5 \% \mathrm{~B}$ at $11 \mathrm{~min}$ to re-equilibrate. The total run time was 12 min per sample. An Agilent technologies Zorbax Eclipse Plus C18 $2.1 \times 50 \mathrm{~mm}$ column was used for the HP LC separation.

The mass spectrometer was operated in targeted multiple reaction monitoring mode and each urine sample was analysed twice, first, in positive ion mode for drugs and metabolites listed in category 'Positive' in table 1 and then in negative ion mode for those listed under 'Negative' in table 1. A total of 40 antihypertensive medications or their metabolites were screened for in urine (table 1).

Total non-adherence to antihypertensive treatment was defined as complete absence of any prescribed antihypertensive medications (or their metabolites where appropriate) in a spot urine sample on screening. Patients whose urine analysis confirmed the presence of fewer medications than prescribed were classified as partially non-adherent.

The project was approved by the University Hospitals of Leicester as an audit project (audit registration number: 5944). Prior to screening, the patients were advised that their urine sample would be assayed for presence of BP lowering drugs. As a part of clinical care, the results of biochemical screening for non-adherence to antihypertensive treatment were reported to the patients (usually during their next appointment in the clinic) and information on the results from HP LC-MS/MS urine-based analysis was included in the clinical letter to patients' general practitioners.

\section{Statistical analysis}

Data are presented as counts/percentages, medians with $25 \%-75 \%$ IQR or means and SDs.

Analysis of association between BP and non-adherence to antihypertensive treatment was conducted using multiple linear regression models with $\mathrm{BP}$ as dependent quantitative variable and age, sex, ethnicity, clinical category (new referrals, follow-up patients, referrals for renal denervation) and therapeutic non-adherence (categorised into binary phenotype) as independent parameters.
Further analyses of association between BP and quantitative measures of non-adherence to treatment were carried out using multiple linear regression models with BP as dependent quantitative variable and age, sex, ethnicity, clinical category (new referrals, follow-up patients, referrals for renal denervation) and either the numerical difference or the ratio of detected/prescribed antihypertensive medications as independent parameters included in the regression models.

All analyses were conducted in STATA package.

\section{RESULTS}

The clinical characteristics of hypertensive patients are shown in table 2 .

Overall, 25\% patients were totally or partially non-adherent to antihypertensive treatment (total non-adherence 10.1\%, partial non-adherence $14.9 \%$ ) (table 3 ). The average number of antihypertensive drugs detected on screening (median 2 (25\%-75\% IQR: 1-3)) was lower than the number of drugs prescribed and screened for (median 3 (25\%-75\% IQR: 2-4)) (table 3). Across the three groups of patients, the highest percentage of total nonadherence with antihypertensive treatment was among those referred for renal denervation-almost one in four patients from this group had none of the prescribed medications detected in urine, that is, complete non-adherence (table 3). The prevalence of complete non-adherence among new referrals to the clinic (Group A) and follow-up patients (Group B) were lower at $8.8 \%$ and $9.1 \%$ (table 2). However, their overall non-adherence

Table 3 Non-adherence to antihypertensive treatment among hypertensive patients

\begin{tabular}{lllll}
\hline Measure of non-adherence & All & Group A & Group B & Group C \\
\hline $\mathrm{N}$ & 208 & 125 & 66 & 17 \\
$\begin{array}{l}\text { Average number of screened } \\
\text { medications }\end{array}$ & $3(2-4)$ & $3(1-4)$ & $4(3-5)$ & $3(2-5)$ \\
$\begin{array}{l}\text { Average number of medications } \\
\text { detected }\end{array}$ & $2(1-3)$ & $2(1-3)$ & $3(1-4)$ & $2(1-3)$ \\
$\begin{array}{l}\text { Complete non-adherence } \\
\text { Partial non-adherence }\end{array}$ & $21(10.1)$ & $11(8.8)$ & $6(9.1)$ & $4(23.5)$ \\
Any non-adherence & $31(14.9)$ & $12(9.6)$ & $19(28.8)$ & - \\
\hline
\end{tabular}

Data are counts and percentages or medians and 25\%-75\% IQR (in brackets). 
Table 4 Association between blood pressures and non-adherence to antihypertensive treatment among hypertensive patients

\begin{tabular}{|c|c|c|c|c|c|c|c|}
\hline Blood pressure & Adherent & Any non-adherent & Complete non-adherent & Beta $(\mathrm{SE})^{*}$ & p Value* & Beta (SE)† & p Value $†$ \\
\hline Clinic SBP $\ddagger$ & $161 \pm 24.0$ & $170 \pm 24.7$ & $177 \pm 28.5$ & $-9(3.7)$ & 0.0209 & $-18(5.4)$ & 0.0010 \\
\hline Clinic DBP $\ddagger$ & $90 \pm 14.4$ & $100 \pm 19.1$ & $107 \pm 18.3$ & $-9(2.4)$ & 0.0003 & $-16(3.5)$ & $1.0 \times 10^{-5}$ \\
\hline $24 \mathrm{~h}$ daytime SBP§ & $152 \pm 19.8$ & $159 \pm 21.1$ & $165 \pm 17.3$ & $-6(4.2)$ & 0.1814 & $-14(5.5)$ & 0.0146 \\
\hline $24 \mathrm{~h}$ daytime DBP§ & $86 \pm 13.1$ & $94 \pm 13.0$ & $100 \pm 9.8$ & $-6(2.6)$ & 0.0286 & $-11(3.2)$ & 0.0006 \\
\hline
\end{tabular}

*Difference between adherence and any non-adherence (both partial and complete) after adjustment for age, sex, ethnicity and clinical category.

tDifference between adherence and complete non-adherence after adjustment for age, sex, ethnicity and clinical category.

¥Systolic blood pressure (SBP) and diastolic blood pressure (DBP) - information available for 152 adherent, 52 any non-adherent and 21 completely non-adherent patients.

$\$ 24 \mathrm{~h}$ daytime SBP and DBP-information available for 121 adherent, 26 any non-adherent and 15 completely non-adherent patients, data in columns $2-4$ are means and SDs of absolute blood pressure values recorded by clinic measurements and $24 \mathrm{~h}$ ambulatory blood pressure monitoring. Beta- $\beta$-coefficient, $\mathrm{p}$ value-level of statistical significance; all from adjusted linear regression models with blood pressure as dependent quantitative variable and age, sex, ethnicity and clinical category (new referrals, follow-up patients, referrals for renal denervation) as well as non-adherence to antihypertensive treatment as independent parameters included in the model.

(complete and partial non-adherence) was calculated at $18.4 \%$ and 37.9\% (Groups A and B, respectively).

After adjustment for age, sex, ethnicity and clinical category (new referrals, follow-up patients, referrals for renal denervation), clinic systolic blood pressure (SBP) and diastolic blood pressure (DBP) as well as $24 \mathrm{~h}$ mean daytime DBP were higher in non-adherent (either partially or completely) than fully adherent patients (table 4). These differences were even more striking when contrasting fully adherent against totally nonadherent patients (table 4). There was a linear relationship between BP and both the numerical difference and the ratio in detected/prescribed medications-those with difference $=0$ and ratio $=1$ (all medications taken and detected) had the lowest clinic SBP, clinic DBP and 24 h mean daytime DBP (figure 1). As a general trend, DBP showed a stronger association with nonadherence to antihypertensive medications than SBP (table 4, figure 1 and figure 2).

\section{DISCUSSION}

Our results revealed that one in four hypertensive patients attending a specialist hypertension centre were partially or totally non-adherent to pharmacological BP lowering therapy on qualitative HP LC-MS/MS urine analysis. We also show that biochemically confirmed non-adherent hypertensive patients have higher BP (in particular DBP) than those who adhere to treatment. Furthermore, the data clearly demonstrate an association between the degree of non-adherence to antihypertensive treatment and clinic as well as $24 \mathrm{~h}$ daytime DBP values-the higher the numerical difference (or lower the ratio) between biochemically detected and prescribed medications, the higher the BP. Finally, we showed that almost one in four patients referred for renal denervation was completely non-adherent to prescribed BP lowering treatment.

Our study is one of the first to systematically use a robust detection method to report the prevalence of non-adherence to antihypertensive therapy among all new referrals to the specialist hypertension centre. Almost one in four of these patients showed some degree of non-adherence and one in 10 were completely non-adherent to BP lowering therapy. A majority of these patients in any secondary/tertiary care centre would routinely undergo many additional tests and procedures in search of the explanation for their apparent unresponsiveness to standard therapy prescribed by primary care. Our data suggest that in $20 \%$ of such patients, these investigations (along with follow-up appointments and exposure to unnecessary additional treatment) could be potentially avoided if HP LC-MS/MS urine analysis was used as a routine screening for non-adherence.
Based on a similar methodology, a recently published survey of 76 hypertensive patients with treatment resistance referred from primary care to an out-patient nephrology centre showed higher $(\approx 50 \%)$ rate of non-adherence to BP lowering therapy. ${ }^{12}$ This difference may be explained by the different inclusion criteria between both studies. Indeed, Jung et $a l^{12}$ screened only patients with resistant hypertension while a majority of patients included in this study were consecutive, unselected referrals from primary care (Group A). The data from our investigation are based on a larger and clinically more diverse sample of hypertensive patients with a wider range of reasons for referral to the clinic. This better reflects the true clinical spectrum of patient population in a specialist hypertension centre. We should also note the apparent difference in the average number of prescribed antihypertensive medications between Jung et al and our study (five vs three). The higher number of BP lowering agents may also explain (at least to some extent) the higher rate of non-adherence in the study by Jung et al given that complex regime of prescribed medications is a recognised risk factor of non-adherent behaviour. ${ }^{13}$

To the best of our knowledge, we are the first to report the rate of non-adherence to antihypertensive treatment among patients referred for renal denervation. What is striking about our findings in this group is their average rate of complete nonadherence. Although non-adherence is a recognised cause of resistant hypertension, ${ }^{2}$ the rates of true non-adherence to antihypertensive therapy were either not reported or reported suboptimally in clinical trials on this group of patients. ${ }^{14-16}$ The data collected in our audit suggest that as many as one in four patients referred for renal denervation would not have qualified for the procedure according to existing guidance on its use which excludes non-adherence to therapy. ${ }^{17} 18$ Of course, it could be argued that renal denervation could be the ideal treatment for persistently non-adherent patients given their lack of acceptance for regular administration of antihypertensive pills. However, at present we would not recommend that renal denervation is offered to non-adherent hypertensive patients. First, currently there is no evidence for the clinical benefits of this intervention in therapeutically non-adherent patients. Second, the existing data suggest that renal denervation may reduce the number of antihypertensive medications but unlikely to cure hypertension completely and as such may not be attractive enough option for patients who choose not to take any $\mathrm{BP}$ lowering medications (ie, all non-adherent patients in our renal denervation group). Third, in a substantial proportion of patients, non-adherence may be driven by fear of and/or certain beliefs around potential side effects of treatment. While stopping unwanted/unacceptable BP lowering medication is usually 
Figure 1 Association between blood pressures and the numerical difference between detected and prescribed antihypertensive medications in all hypertensive patients. Point data are absolute blood pressure values recorded on either clinic or $24 \mathrm{~h}$ ambulatory monitoring, $\mathrm{p}$ valueadjusted (for age, sex, ethnicity and clinical category (new referrals, follow-up patients, referrals for renal denervation)) level of statistical significance for every unit change in blood pressure per unit change in the difference between detected and prescribed antihypertensive medications.
Figure 2 Association between blood pressures and the numerical ratio of detected and prescribed antihypertensive medications in all hypertensive patients. Point data are absolute blood pressure values recorded on either clinic or $24 \mathrm{~h}$ ambulatory monitoring, $\mathrm{p}$ valueadjusted (for age, sex, ethnicity and clinical category (new referrals, follow-up patients, referrals for renal denervation)) level of statistical significance for every unit change in blood pressure per unit change in the ratio of detected and prescribed antihypertensive medications.
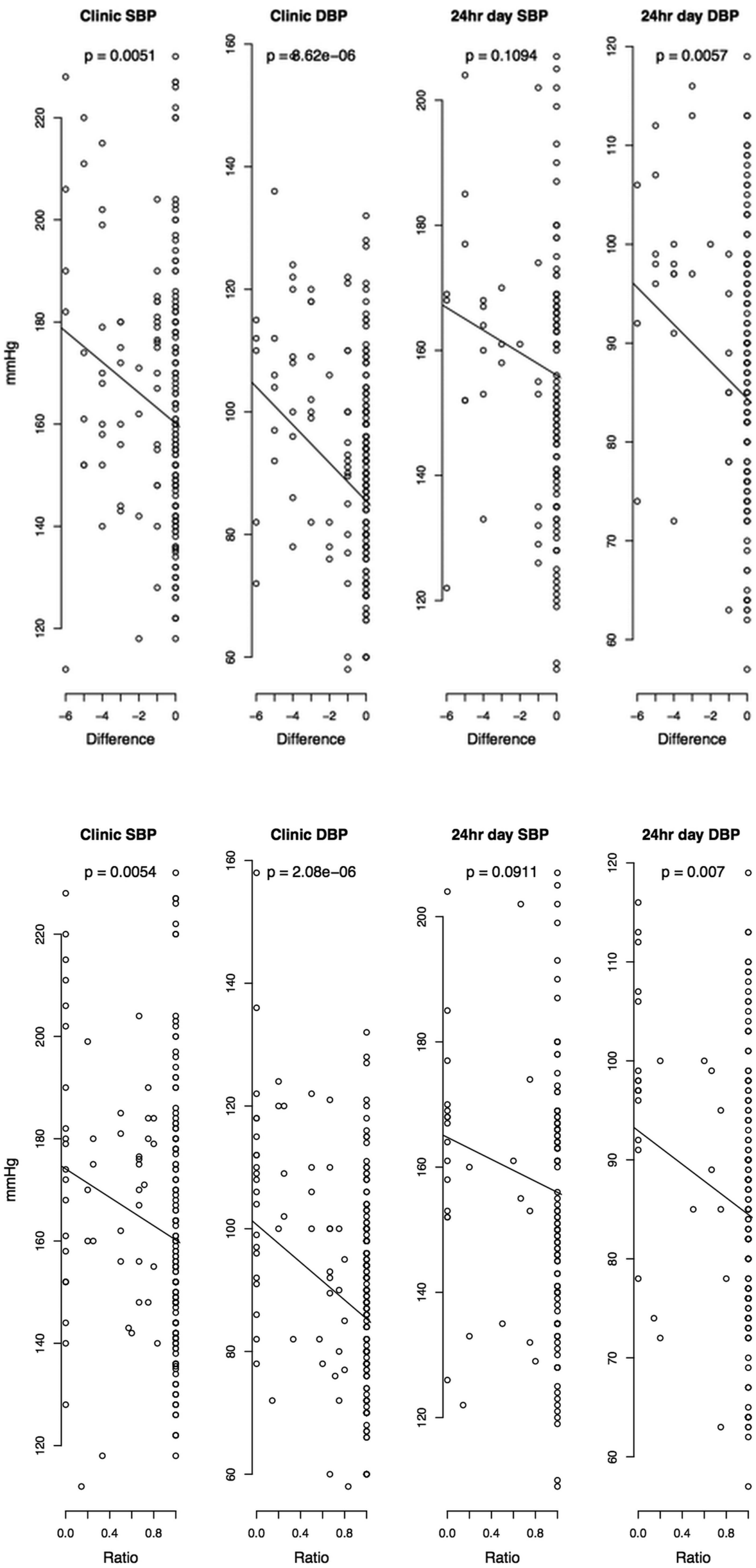
sufficient to eliminate these perceptions, renal denervation while generally safe is irreversible. Therefore, it may be difficult to convince such non-adherent patients and/or their managing clinicians to consider this mode of treatment.

Our study has several limitations. First, information on $24 \mathrm{~h}$ ambulatory BP lowering was not available for approximately $25 \%$ of included patients. The lower number of observations (and thus lower power to detect association) probably explains somewhat the weaker relationship between non-adherence to treatment and $24 \mathrm{~h}$ daytime BP values when compared with clinic BP measurements. Second, the group of patients referred for renal denervation was small. We also acknowledge several imperfections of the method used as a screening of adherence to treatment; that is, a single spot urine analysis may not fully account for periodicity (both personal and seasonal) ${ }^{7}$ of nonadherence to treatment. Furthermore, intuitively, one might expect some patients to better adhere to treatment on the day of clinic attendance (the so-called 'tooth brush effect ${ }^{\text {'19 }}$ ), given that poor BP control in clinic is likely to prompt further investigation and/or treatment escalation. Thus, the spot urine assay may underestimate chronic or intermittent non-adherence. However, this limitation applies to the majority of currently used adherence tests conducted in the clinic prior to appointments. Repeated urine tests could provide a better insight into patients' adherence to antihypertensive therapy. We also recognise the unavailability of indirect measures of adherence to antihypertensive treatment in this project. Further studies on utility and cost effectiveness of HP LC-MS/MS urine analysis should be conducted against indirect measures of adherence to inform future health policies and clinical practice. Finally, this analysis was conducted as an audit of adherence and we acknowledge the risks of potential bias from unmeasured confounders in an observational study.

However, biochemical screening for adherence to antihypertensive treatment using a spot urine sample has several major advantages. First, it is a completely non-invasive procedure that can be conducted by a healthcare assistant prior to routine clinical appointments. Unlike many other previously used methods of screening, the HP LC-MS/MS analysis provides a clear 'Yes/No' answer to a question on presence/absence of antihypertensive medications based on direct measurement of urine. Although the costs of HP LC-MS/MS analyser are not trivial $(\approx £ 150000)$, many major clinical centres have access to such technology, a single urine test is relatively inexpensive (approximately $£ 30$ ) and the results can be provided quickly as sample processing takes about $3 \mathrm{~h}$. Moreover, the frozen samples are stable when stored prior to biochemical analysis. Finally, HP LC-MS/MS is a recognised method with good to excellent sensitivity and specificity to detect many pharmacological agents in urine. ${ }^{20-22}$

\section{CONCLUSIONS}

In summary, our data provide a unique insight into the prevalence of non-adherence to antihypertensive treatment using an objective and direct detection of BP lowering medications in urine by HP LC-MS/MS. We show that a significant proportion of patients in a specialist centre show at least some degree of treatment non-adherence and that their BP levels correlate well with the degree on non-adherence. Finally, within the constraints of a small sample size, we show for the first time alarmingly high levels of complete biochemical non-adherence to treatment among patients referred for renal denervation. Future large multi-centre studies will be needed to provide estimates of direct biochemical non-adherence to treatment in other populations and evaluate the cost effectiveness of this screening method in the context of overall expenditure for management of resistant hypertension. Further investigations should also examine if discussing the results of HPLC-MS/MS-based screening with patients has a potential to improve their adherence to antihypertensive treatment and, ultimately, BP control.

\section{Key messages}

What is already known on this subject?

Non-adherence to antihypertensive treatment is a recognised cause of poor blood pressure control and 'pseudo-resistant' hypertension.

\section{What this study adds?}

Our data show that approximately one in four patients in a specialist hypertension clinic is at least partially non-adherent to blood pressure lowering treatment. We are the first to report the high prevalence of directly measured non-adherence to antihypertensive treatment in a group of patients referred for renal denervation.

How might this impact on clinical practice? Screening for non-adherence to antihypertensive treatment by high-performance liquid chromatography-tandem mass spectrometry-based urine analysis is a simple, non-invasive diagnostic test with a potential to better stratify patients prior to treatment escalations and expensive and irreversible procedures such as renal denervation.

Contributors All authors of the paper fulfil the criteria of authorship. Conception and design of the study: MT, PP, NJS, WM, AS, BW. Analysis and interpretation of data: MT, CW, PP, NM, RD, JH, PG, AS, BW. Drafting the manuscript: MT, BW. Critical revision of manuscript for intellectual content: CW, PP, NM, RD, JH, NJS, PG, WM, AS. Final approval of the manuscript: MT, CW, PP, NM, RD, JH, NJS, PG, WM, AS, BW

Competing interests MT is supported by British Heart Foundation. BW is a National Institute for Health Research Senior Investigator and his research is supported by the National Institute for Health Research University College London Hospitals Biomedical Research Centre. NJS holds a Chair funded by British Heart Foundation and is a National Institute for Health Research Senior Investigator. CW NM and NJS are supported by the National Institute for Health Research Leicester Cardiovascular Biomedical Research Unit and this work is part of the portfolio of research undertaken by the Unit.

Provenance and peer review Not commissioned; externally peer reviewed.

Open Access This is an Open Access article distributed in accordance with the Creative Commons Attribution Non Commercial (CC BY-NC 3.0) license, which permits others to distribute, remix, adapt, build upon this work non-commercially, and license their derivative works on different terms, provided the original work is properly cited and the use is non-commercial. See: http://creativecommons.org/ licenses/by-nc/3.0/

\section{REFERENCES}

1 Steiner JF. Rethinking adherence. Ann Intern Med 2012;157:580-5.

2 Myat A, Redwood SR, Qureshi AC, et al. Resistant hypertension. BMJ 2012;345: e7473.

3 Myat A, Redwood SR, Qureshi AC, et al. Renal sympathetic denervation therapy for resistant hypertension: a contemporary synopsis and future implications. Circ Cardiovasc Interv 2013;6:184-97.

4 Perreault $S, Y u$ AY, Côté $R$, et al. Adherence to antihypertensive agents after ischemic stroke and risk of cardiovascular outcomes. Neurology 2012;79:2037-43.

5 Corrao G, Parodi A, Nicotra F, et al. Better compliance to antihypertensive medications reduces cardiovascular risk. J Hypertens 2011;29:610-18.

6 Mazzaglia G, Ambrosioni E, Alacqua M, et al. Adherence to antihypertensive medications and cardiovascular morbidity among newly diagnosed hypertensive patients. Circulation 2009;120:1598-605. 
7 Vrijens B, Vincze G, Kristanto $P$, et al. Adherence to prescribed antihypertensive drug treatments: longitudinal study of electronically compiled dosing histories. BMJ 2008;336:1114-17.

8 Tomaszewski M, Lacka B, Zukowska-Szczechowska E, et al. The evaluation of hypertensive patients' adherence to the pharmacological treatment of hypertension. Wiad Lek 1999;52:573-80.

9 Chobanian AV. Impact of nonadherence to antihypertensive therapy. Circulation 2009;120:1558-660.

10 Santschi V, Rodondi N, Bugnon O, et al. Impact of electronic monitoring of drug adherence on blood pressure control in primary care: a cluster 12-month randomised controlled study. Eur J Intern Med 2008;19:427-34.

11 van Onzenoort HA, Verberk WJ, Kroon AA, et al. Electronic monitoring of adherence, treatment of hypertension, and blood pressure control. Am J Hypertens 2012;25:54-9.

12 Jung 0 , Gechter JL, Wunder C, et al. Resistant hypertension? Assessment of adherence by toxicological urine analysis. J Hypertens 2013;31:766-74.

13 Nuesch R, Schroeder K, Dieterle T, et al. Relation between insufficient response to antihypertensive treatment and poor compliance with treatment: a prospective case-control study. BMJ 2001;323:142-6.

14 Persu A, Renkin J, Thijs $L$, et al. Renal denervation: ultima ratio or standard in treatment-resistant hypertension. Hypertension 2012;60:596-606.
15 Krum H, Schlaich M, Whitbourn R, et al. Catheter-based renal sympathetic denervation for resistant hypertension: a multicentre safety and proof-of-principle cohort study. Lancet 2009;373:1275-81.

16 Symplicity HTN-2 Investigators. Renal sympathetic denervation in patients with treatment-resistant hypertension (The Symplicity HTN-2 Trial): a randomised controlled trial. Lancet 2010;376:1903-9.

17 Schmieder RE, Redon J, Grassi G, et al. ESH position paper: renal denervation-an interventional therapy of resistant hypertension. J Hypertens 2012;30:837-41.

18 Mahfoud F, Lüscher TF, Andersson B, et al. Expert consensus document from the European Society of Cardiology on catheter-based renal denervation. Eur Heart J 2013;34:2149-57.

19 Chatterjee JS. From compliance to concordance in diabetes. J Med Ethics. 2006;32:507-10

20 Fitzgerald RL, Rivera JD, Herold DA. Broad spectrum drug identification directly from urine, using liquid chromatography-tandem mass spectrometry. Clin Chem 1999:45:1224-34.

21 Murray GJ, Danaceau JP. Simultaneous extraction and screening of diuretics, beta-blockers, selected stimulants and steroids in human urine by HPLC-MS/MS and UPLC-MS/MS. J Chromatogr B Analyt Technol Biomed Life Sci 2009;877:3857-64.

22 Mazzarino $\mathrm{M}$, de la Torre $\mathrm{X}$, Botrè $\mathrm{F}$. A screening method for the simultaneous detection of glucocorticoids, diuretics, stimulants, anti-oestrogens, beta-adrenergic drugs and anabolic steroids in human urine by LC-ESI-MS/MS. Anal Bioanal Chem 2008:392:681-98. 